\title{
Evaluation préliminaire d'un dispositif de coévaluation en travaux pratiques d'endodontie
}

\author{
Franck DIEMER, Marie-Eve LAURET, Bodie PRATS, Paul CALAS*
}

Résumé Contexte : L'aménagement des voies d'accès première étape du traitement endodontique, obét à des critères préds reconnus uni versell lement. U nestratégi e de coévaluation a éééintégrée dans l'ensignement pratique de cette disciplineà la faculté de chirurgi e dentaire deToulouse B uts : L'objectif de cette étude était de savoir s' la coévaluation de l'amé nagement des voi es d'accès en dodontiquesà partir de critères définis et objecti vés visuellement pouvait permettreà l'étudiant d'obtenir un acte professionnellement satisfaisant. Matériel et méthodes: Cette étude porte sur l'un des objectifs de l'enseignement pratique d'en dodontie de troisième année (l'aménagement des voies d'accès) pendant trois années universitaires consécutives Une fiche d'évaluation est mise au point et un support visuel permet aux éudiants d'objectiver les différents critères d'évaluation. L'analyse des résultats expl oite les scores, la moyenne des scores desétudiants, la distance à l'évaluateur et le taux d'évaluation correcte. Un quetionnaire de fin d'année tente d'évaluer la perception des étudiants pour cet outil pédagogique. Résultats: Les scores progressent au cours des séances. Les étudiants apprennent à sévaluer en trois à quatre séances Ils aimerai ent voir cette coévaluation appliquée à l'ensemble de notre enseignement. Conclusi on : La coévaluation de l'aménagement des voi es d'accès en dodontiques à partir de critères définis et objectivés visuellement, semble aider l'étudiant à développer des compétences pour obtenir un acte professionnellement satifaisant.

Mot clés Endodontie; travaux pratiques; coévaluation ; rétroaction ; progression ; aménagement de voie d'accès.

Abstract context: Root canal access preparation which is the first step to endodontic treatment, obeys universally recogni sed precise criteria. A strategy of coevaluation has been integrated into the practical teaching of this subject at the dental surgery school in Toulouse. Aim: To assess wether the coevalution of the preparation of the endodontic root canal access from defined and visually objectified criteria could help the sudent to perform a professionally satifactory treatment. Material and method: this study is about one of the objectives of third year endodontic practical teaching and learning (preparation of root canal access) during three consecutive university years An evaluation form has been developed and a visual support helps students to objectify different evaluation criteria. Processing of results makes use of marks, student ave rage marks, distance to the evaluator and ratio of correct evaluation. A questionnaire at the end of theyear tries to evaluate the student perception of thisteaching tool. Results: marks improve over the sessions. Students learn how to evaluate themselves within three or four sessions. They would like to see this coeval uation applied to other courses Conclusion: The coe valuation of the preparation of the endodontic root canal access from defined and visually objectified criteria could help the student to perform a professi onally satisfactory treatment.

Key Words Endodontics; practical assignments; coevaluation; feed back; progression; root canal access.

Pédagogie M édicale 2005 ; 6 : 79-87

\footnotetext{
*Faculté de Chirurgie Dentaire de Toulouse-Service d'Odontologie Conservatrice - Endodontie Correspondance: Franck Diemer - Facultéde chirurgie dentaire de Toulouse - 3 chemin des Maraîchers - 31062 Toulouse Cedex Téléphone : 0562489723 - mailto: franck.diemer@wanadoo.fr
} 


\section{Concepts et Innovations}

\section{Introduction}

L'endodontie ou science du traitement de la pulpe dentaire - saine ou pathologique - fait partie de l'enseignement dispensé aux étudiants en chirurgie dentaire. A la faculté de Toulouse, l'apprentissage de cette discipline reconnue difficile commence dès la troisième année d'études (D 1).

Afin d'améliorer l'efficience de notre enseignement, nous avons impliqué l'étudiant dans sa formation. Un nouveau dispositif pédagogique aété mis en place, intégrant en particulier une coévaluation des travaux pratiques. Le terme de coévaluation reconnaît différentes acceptions : pour certains, il désigne le partage de l'évaluation des apprentissages par des enseignants partenaires (enseignant acadé miqueet maître de stage en entreprise, par exemple) ; pour d'autres, il fait référence à l'évaluation par les pairs ; dans cetravail, nous l'entendrons comme désignant la conjonction d'une évaluation formative par l'enseignant et d'une autoévaluation par l'étudiant ${ }^{1}$. L'autoévaluation est un outil pédagogique relativement ancien ${ }^{2}$, modélisé depuis $1984^{2}$ dans l'enseignement général et depuis $1998^{3}$ dans les formations professionnalisantes (infirmières, enseignants). D ans la littérature odontologique, et notamment dans notre discipline - I'odontologie conservatrice ondodontie $(O C E)$ - cet outil, bien que peu mentionné, a déjà éééutilisé. Ainsi, dans un article récent ${ }^{4}$ une stratégie d'autoévaluation avec support visuel a été utilisée par les auteurs spécialistes en O CE : l'étudiant expose à un tuteur ce qu'il pourrait faire pour modifier ses erreurs. D'une manière générale, il est tenu pour acquisque l'autoévaluation favorise la métacognition, caractéristique essentielle dans la perspective du développement des compétences 5 . Cette étude porte sur l'évaluation préliminaire de ce dispositif, en formation initiale à l'endodontie. $\mathrm{N}$ ous avons choisi, comme support d'étude, l'aménagement des voies d'accès endodontiques car cette étape obét à des critères précis reconnus universellement. Après l'exposé de la problématique à laquelle répond ce travail d'évaluation, nous décrirons le dispositif mis en place et les résultats obtenus sur trois années consécutives.

\section{Problématique}

\section{Contexte professionnel}

A la difficulté intrinsèque de l'acte endodontique et de la gestion globale du patient, s'ajoutent des obstacles techniques supplémentaires, tels l'ouverture buccale du patient ou l'encombrement de nos instruments. Ils obligent bien souvent le professionnel à travailler en vision indirecte dans un miroir ou à l'aveugle. $C e s$ techniques demandent un long apprentissage afin de parvenir à mấtriser ses gestes dans de telles situations. Le statut de profession libérale fait du chirurgien dentiste un travailleur indépendant, seul juge de son travail. De plus, aucune obligation de résultats n'est requise dans le code de déontologie. Cette situation impose au chirurgien-dentiste d'être en mesure de développer des stratégies d'autoévaluation ${ }^{6}$, qui sont classiquement peu sollicitées en forma tion initiale.

\section{Eléments descriptifs de la formation initiale}

L'enseignement de l'endodontie est formalisé par des objectifs. Ces objectifs sont de trois ordres et concernent la connaissance du matériel et des matériaux nécessaires à la réalisation d'un acte endodontique, celle de l'anatomie dentaire et des variations possibles et enfin la connaissance des différentes étapes du traitement des pathologies pulpaires et celles associées aux tissus de soutien de la dent. Au cours de chaque séance de travaux pratiques, l'éudiant met en avant ses connaissances théoriques pour réaliser le travail demandé.

Avant la mise en place de ce nouveau dispositif pédagogique, l'acte endodontique était évalué dans sa globalité. La difficulté du traitement croissait en cours d'année avec I'utilisation de dents à l'anatomie de plus en plus complexe. Cette approche ne permettait pas aux éudiants de réaliser à quelle étape du soin ils avaient des problèmes et empêchait d'optimiser leur progression.

Depuis 1999, nous avons mis en place de nouvelles méthodes d'enseignement. Chaque étape de l'acte endodontique fait l'objet d'un apprentissage spécifique. Ainsi, quatre temps opératoires ont été individualisés avec des objectifs précis:

1- I'aménagement des voies d'accès (cavité précise permettant d'accéder au réseau pulpaire - tissus conjonctif et vasculo-nerveux appelé communément «nerf »);

2- le cathétérisme (pénétration initiale) et la mesure de la longueur de travail (hauteur de la dent) ;

3- le nettoyage et la mise en forme des canaux (élargissement) ;

4- enfin l'obturation (fermeture étanche du réseau cana laire à l'aide de biomatériaux : ciment et gutta percha).

N ous avons fait I'hypothèse qu'un « feed-back » informatif 
associé à un dispositif d'autoévaluation permettrait aux étudiants d'évoluer de manière harmonieuse et efficace dans leurs apprentissages. II sagit d'un principe d'évaluation formative qui a déjà fait ses preuves ${ }^{5}$. Pour améliorer la précision de ce retour informatif, il est nécessaire de déterminer des critères de jugement précis et pertinents?. Une note d'information globale sur le travail réalisé, avec une grille de correction, explique point par point l'évaluation de l'enseignant.

L'utilisation d'un support visuel ${ }^{7}$ peut favoriser la compréhension du travail attendu. L'association à un dispositif d'autoévaluation ${ }^{7,8}$ faciliterait I'objectivation deserreurs commises.

Ainsi, nous avons supposé qu'un système de coévaluation ${ }^{2}$ permet aux apprentis de se rendre compte de leurs erreurs et de progresser dans leur pratique ${ }^{9,10}$. Pour tester cette hypothèsenous avons travaillé sur une situation bien définie : I'aménagement des voies d'accès sur trois années consécutives. II sagit de la première étape lors d'un acte endodontie. II permet d'accéder au réseau pulpaire, avant les actes chirurgicaux proprement dits (cathétérisme, mesure de la longueur de travail, nettoyage et mise en forme des canaux, puis obturation).

N ous avons cherché à savoir si la coévaluation de l'aménagement des voies d'accès endodontiques à partir de critères définis et objectivés visuellement peut permettre à l'étudiant d'obtenir un acte professionnellement satisfaisant.

\section{Matériel et méthodes}

\section{Etudiants - 0 bjectifs de travail}

L'étude a été réalisée au cours des travaux pratiques d'endodontie de troisième année. Elle porte sur trois années universitaires consécutives, au cours desquelles le nombre d'étudiants de chaque promotion a été différent (respectivement 57,55 et 58 ).

Les objectifs concernant l'aménagement des voies d'accès doivent être validés par tous les étudiants. La première année de mise en place du dispositif (1999-2000), seules les voies d'accès sur prémolaires, trois au total, ont ééééva luées. Les années suivantes (2000 à 2002), nous avons élargi l'éude à l'ensemble des dents traitées (7 dents au total). Au fur et à mesure de leur réalisation, toutes les voies d'accès sont éval uées par l'enseignant et lesétudiants.

\section{Fiche d'éval uation}

Une fiche d'évaluation a été conçue en retenant six paramètres: la forme de la cavité, sa dimension, sa position par rapport au type de dent traitée, l'absence d'interférence (interaction entre un instrument de cathétérisme et une paroi de la cavité), la dépouille (conicité) et le respect du plancher (zone inter-radiculaire sur les prémolaires et les molaires) (T ableau 1). Pour les trois premiers paramètres il faut statuer sur la proposition «j'ai bien atteint l'objectif »

\section{Tableau 1 :}

Fiche d'évaluation utilisée pour apprécier les aménagements des voies d'accès par les étudiants et grille de correction de l'enseignant

\begin{tabular}{l|c|c|c|c}
$\begin{array}{l}\text { Forme extérieure } \\
\text { de la cavité d'accès }\end{array}$ & $\begin{array}{c}\text { Tout à fait } \\
\text { d'accord }\end{array}$ & $\begin{array}{c}\text { Plutôt } \\
\text { d'accord }\end{array}$ & $\begin{array}{c}\text { Plutôt } \\
\text { pas d'accord }\end{array}$ & $\begin{array}{c}\text { Pas du tout } \\
\text { d'accord }\end{array}$ \\
\hline Les contours sont réguliers & 4 & 2 & 0 & -2 \\
Les dimensions de la cavité sont correctes & 4 & 2 & 0 & -2 \\
L'ouverture est centrée & 2 & 1 & 0 & -1
\end{tabular}

Accès aux canaux

La voie d'accès est conique

II n'y a pas d'interférence

II n'y a pas de marche, le plancher n'est pas fraisé

\begin{tabular}{|c|c} 
OUI & NON \\
4 & 0 \\
2 & 0 \\
4 & 0
\end{tabular}




\section{Concepts et Innovations}

avec quatre niveaux de réponse : tout à fait d'accord, plutôt d'accord, plutôt pas d'accord et pas du tout d'accord. Pour les trois derniers paramètres d'accessibilité aux canaux, deux niveaux de réponse ont été établis : oui ou non.

Lors de l'autoévaluation, des photographies de cavité sont projetées. Ce système visued d'aide à l'autoévaluation, repris la deuxième et la troisième année, a été affiné au cours de la première année. Chaque paramètre est illustré d'une part, par une photographie montrant la cavité idéale correspondant à l'appréciation « tout à fait d'accord », d'autre part, par deux photographies illustrant respectivement l'erreur par excès et l'erreur par défaut (F igure 1 ).

Les réponses sont par la suite transformées en score, chaque paramètre étant pondéréet affectéd'un coefficient de 1 à - 0,5 en fonction du niveau de satiffaction (T ableau 1).

La même grille de correction, utilisée par l'enseignant et l'étudiant, est donc établie selon le tableau $n^{\circ} 1$. Elle permet d'obtenir un score sur 20, qui n'est pas influencépar la capacité de l'étudiant à s'évaluer. U ne fiche d'évaluation regroupant l'autoévaluation de l'étudiant et celle de l'enseignant est communiquée en début de séance suivante à l'étudiant.

\section{Analyse des résul tats}

L'utilisation de la moyenne des scores des évaluations de chaque voie d'accès et de la répartition de ces scores permet de visualiser le comportement de l'ensemble de la promotion des étudiants. La distance à l'éval uateur et le taux d'évaluation correct rendent compte de la capacité individuelle à l'éval uation. Ces deux indicateurs permettront l'analyse des évaluations de chaque étudiant. La troisième année, l'enseignant a été changé pour évaluer l'influence du formateur. En fin d'année un questionnaire est rempli par l'étudiant afin de mieux comprendre comment il perçoit cet outil pédagogique.

Le faible nombre d'étudiants de D $1(55 \varangle<58)$ au cours des trois années de notre étude ne permet pas de remplir

\section{Figure 1 :}

\section{Copie d'écran de l'illustration du paramètre « dimension » lors d'une séance de correction pour une prémolaire mandibulaire}

\section{Les dimensions sont correctes :}

Tout à fait d'accord

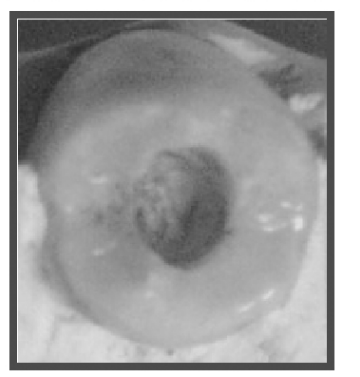

Pas du tout d'accord

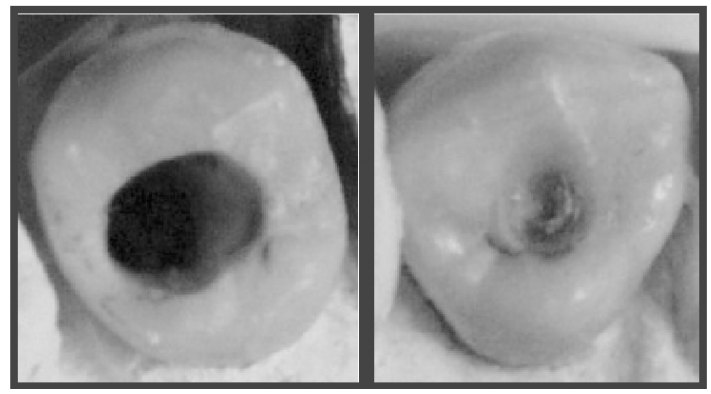

Plutôt d'accord

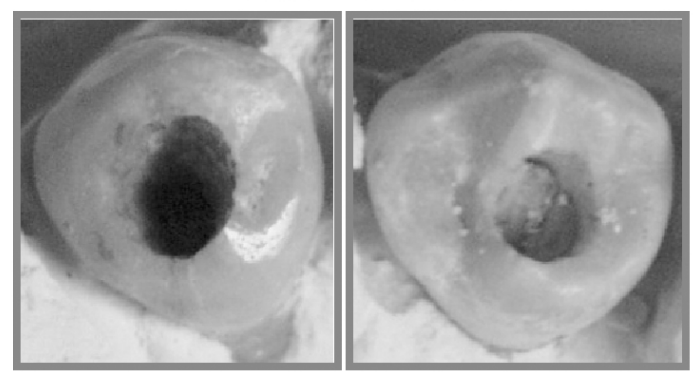

Plutôt pas d'accord

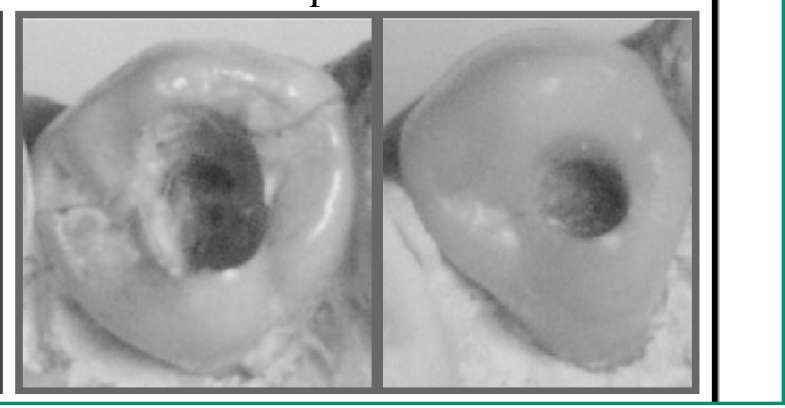


la condition $\mathrm{np}>5$; c'est pourquoi nous avons utilisé le test non paramétrique en séries appariées de Wilcoxon pour notre étude statistique. II a permis d'analyser statistiquement chaque série d'évaluation et mis en évidence l'absence ou l'existence de différence au niveau des cota tions de l'enseignant et des étudiants.

\section{Résultats}

\section{Première année}

La première année d'étude, que nous avons considérée comme préliminaire, a vu les résultats des étudiants progresser d'une valeur médiocre à une valeur correcte avec une convergence entre les apprentis et le formateur. II semble donc que les éudiants aient appris à s'autoévaluer plus justement au fil de l'année. Les critères d'évaluation, au début difficiles à visualiser, ont été assimilés au cours de l'année. L'analyse statistique confirme ces résul- tats. A la question : "Y-a-t-il une différence de notation entre l'enseignant et les étudiants? », le test non paramé trique de Wilcoxon en séries appariées répond affirmativement pour les deux premières dents $(p=0,0002$ dansles deux cas) et négativement pour la dernière $(p=0,4432)$. $N$ ous observons égal ement une amélioration des résultats au fil des séances avec, à la dernière séance d'autoévalua tion, des scores supérieurs à 10 pour plus de $90 \%$ de la première promotion. Cette année préliminaire nousa permis d'affiner la méthode d'évaluation et de proposer aux promotions suivantes une méthode plus explicite et satisfaisante (photographies pour chaque item avec exemple d'erreurs ; évaluation élargie à toutes les cavités d'accès).

\section{D euxième et troisième anné}

Les résultats des deux années suivantes (2000-2002) constituent l'étude proprement dite. Lors des premières séances d'autoévaluation, les étudiants ressentaient des

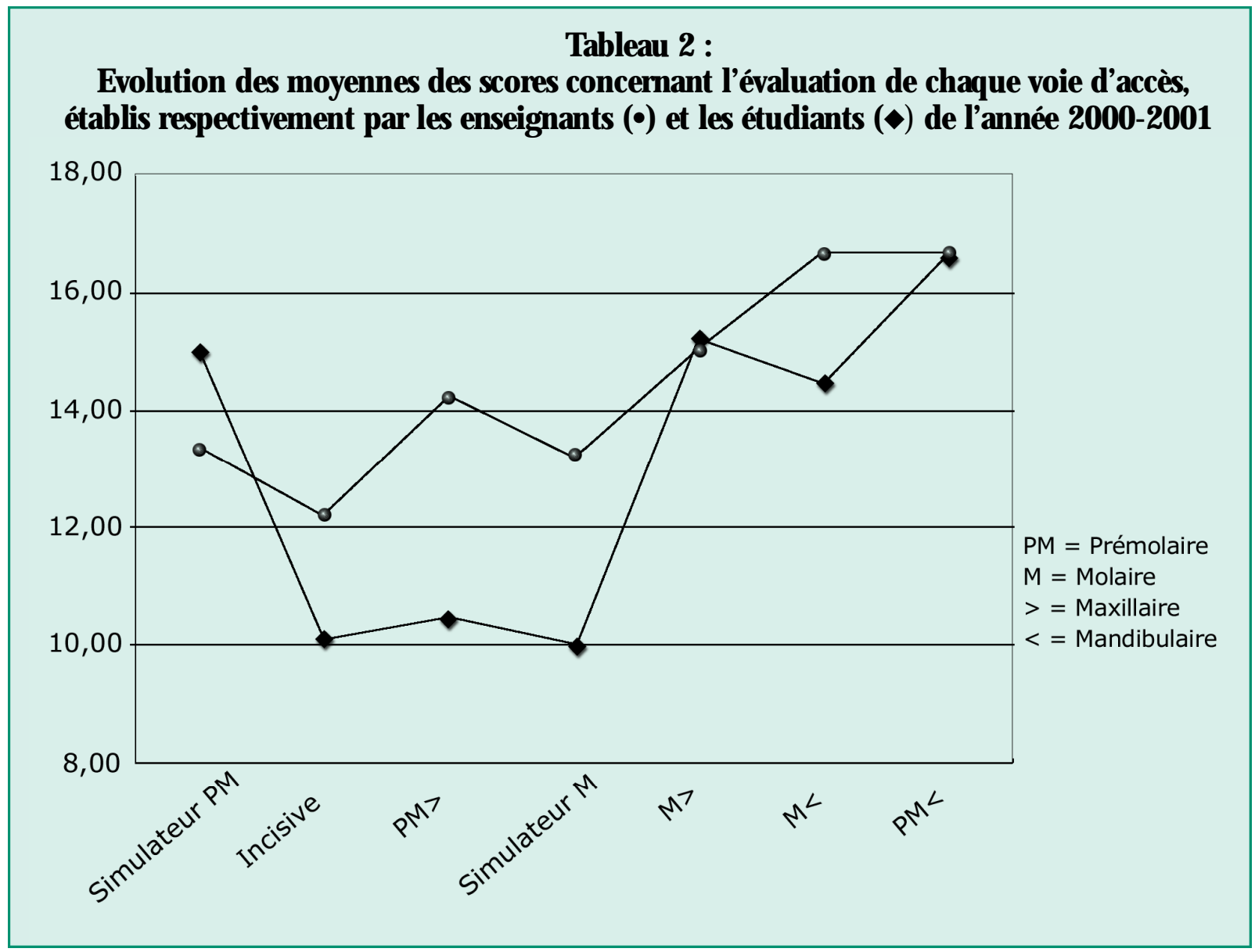




\section{Concepts et Innovations}

\section{Tableau 3 : \\ Evolution des taux d'évaluations correctes (concordance entre l'évaluation de l'étudiant et celle de l'enseignant) au cours de l'année 2000-2001}

\section{Taux d'évaluations correctes}

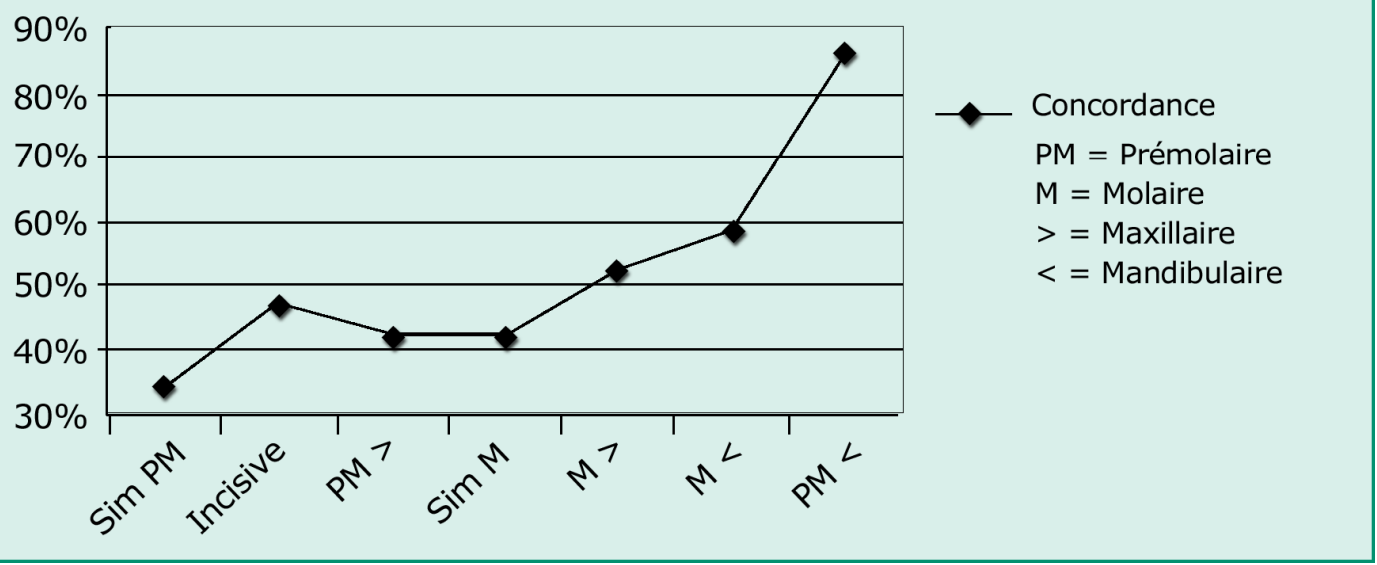

difficultés à sévaluer justement avec des tendances à se sur- ou sous- évaluer. L'analyse statistique montre une différence significative entre le formateur et les étudiants ( $p$ : varie de 0,0001 à 0,0056 ). M ais au fil des séances, les étudiants ont assimilé les critères d'évaluation et nous observons que leur moyenne converge avec celle du formateur (T ableau 2).

La distance à l'évaluateur, représentée par la somme des valeurs absolues des écarts séparant l'évaluation du correcteur de l'évaluation de l'étudiant pour chaque paramètre, tend aussi à diminuer au fil du temps. II semble donc que les étudiants comprennent les critères d'évaluation et arrivent à juger plus précisément leur travail. De la même manière, letaux d'éval uation correcte (rapport du nombre d'évaluations identiques au correcteur sur le nombre de paramètres évalués) progresse (T ableau 3). L'analyse statistique trouve ainsi une différence non significative ( $p=$ 0,1124 à 0,6762) entre enseignant et apprentis pour les dernières cavités à réaliser. Les scores obtenus par ces deux promotions sont également meilleurs au fil du temps. La presque totalité des étudiants obtient en effet un score supérieur à 10 pour la dernière cavité réal isée. Le changement de formateur lors de la troisième année de cette étude ne semble pas affecter ces résultats positifs.

Les résultats de l'audit sur les trois années consécutives donnent les mêmes conclusions. La difficulté ressentie au départ par cette nouvelle méthode samoindrit au fil des séances d'autoévaluation. L'intérêt des étudiants est géné ral et ils paraissent très satisfaits par la méthode. Leur souhait serait que la coévaluation soit appliquée à tous nos objectifs de travaux pratiques.

\section{Discussion}

Cette étude tente d'évaluer la transposition en OCE d'un outil pédagogique, la coéval uation, reconnue efficace dans d'autres formations ${ }^{2}$ 3, 4 . Trois promotions d'étudiants de troisième année ont été observées. Chaque promotion (55 à 58 étudiants) a participé dans son ensemble à cette étude. $D$ ans cette étude exploratoire, I'utilisation d'un groupetémoin n'a pas étéretenue pour éviter une pertede puissance statistique.

Pour évaluer l'approche pédagogique proposée, nous avons sélectionné un objectif de formation (l'aménage ment des voies d'accès) et mesuré à son sujet trois indicateurs (moyenne des scores, taux d'évaluation correcte et distance à l'évaluateur). Lors de la mise au point des séances de correction, durant la première année de notre étude, le diaporama de présentation a évolué A la première séance, nous avions présenté aux étudiants des photographies de cavité d'accès correctes selon les différents paramètres précités. Cette présentation manquait d'indications visuelles car elle ne montrait que la repré sentation idéale du paramètre à évaluer et les explications 


\section{Evaluation préliminaire d'un dispositif de coévaluation en TP...}

del'enseignant étaient de cefait jugées insuffisantes par les étudiants. Cela nous a conduit à faire évoluer la présenta tion des informations destinées au feedback. La deuxième séance proposait des photographies de cavité correctes et incorrectes (exemples d'erreurs). Ces améliorations ont canalisé la correction des étudiants ; le type de questions sest ainsi centré sur certains points récurrents. $M$ ais cela semblait encore insuffisant. L'outil a donc été encore modifié ; à la troisième séance, nous avons proposé aux étudiants une photographie montrant la cavité idéale et répondant à l'item «tout à fait d'accord », complétée par deux photographies illustrant respectivement l'erreur par défaut et celle par excès. Cette séance a permis une correction pratiquement sans question. II semble que l'évolution del'outil ait permis une meilleure compréhension des critères d'évaluation par les étudiants mais également une progression des scores.

Cette dernièreforme de présentation a été utilisée les deux années suivantes. L'ensemble des étudiants a également progressé tout au long de l'année. Ainsi, le fait de rendre explicite les critères de correction nous semble avoir favorisé l'aptitude des étudiants à l'autoévaluation. En toute rigueur, la méthode mise en œuvre dans notre étude ne permet pas d'exclure le fait que cette progression soit tout simplement due à la répétition du geste; les étudiants qui au début de l'année sont novices dans cette discipline acquerraient au cours de l'année une certaine dextérité manuelle. Une étude complémentaire recourant à un groupe témoin sans autoévaluation pourrait apporter à cet égard des arguments additionnels.

Les progressions ne sont pas visibles de la même manière pour tous les étudiants. Certains démontrent dès le début des aptitudes importantes; ils apprennent à s'évaluer très rapidement et la progression de leurs scores est dès lors moins importante. L'essentiel de la promotion apprend à s'évaluer en quatre à six séances. Ces étudiants progressent ensuite très rapidement et deviennent aptes à critiquer leur travail. $N$ ous postulons que I'utilisation de cette capa cité favorisera, au cours de leur exercice libéral, le déve loppement de pratiques caractéristiques du « praticien réflexif ${ }^{11}$.

N ous avons également rencontré des étudiants qui ressentent des difficultés pour comparer leur travail et le support visuel de correction. Leur progression est, de ce fait, peu importante et anarchique. II semble donc important de détecter ces étudiants et leur proposer une autre stratégie d'évaluation formative afin qu'ils comprennent leurs erreurs et progressent. Cette alternative reste à définir.
La progression de nos étudiants nes'est pas faite de façon linéaire. D eux types de support ont été utilisés pour les séances de travaux pratiques (TP) : des simulateurs (prémolaires et molaires) qui permettent une vision directe et donc une exécution plus facile du geste et des dents naturelles, montées sur modèle en plâtre et pour lesquels letravail s'effectue sur « fantôme » (fausse tête avec joues : figure 2). La difficulté réside dans le traitement des dents maxillaires car il oblige l'exécutant à travailler en vision indirecte, en regardant ses gestes dans un micro-miroir.

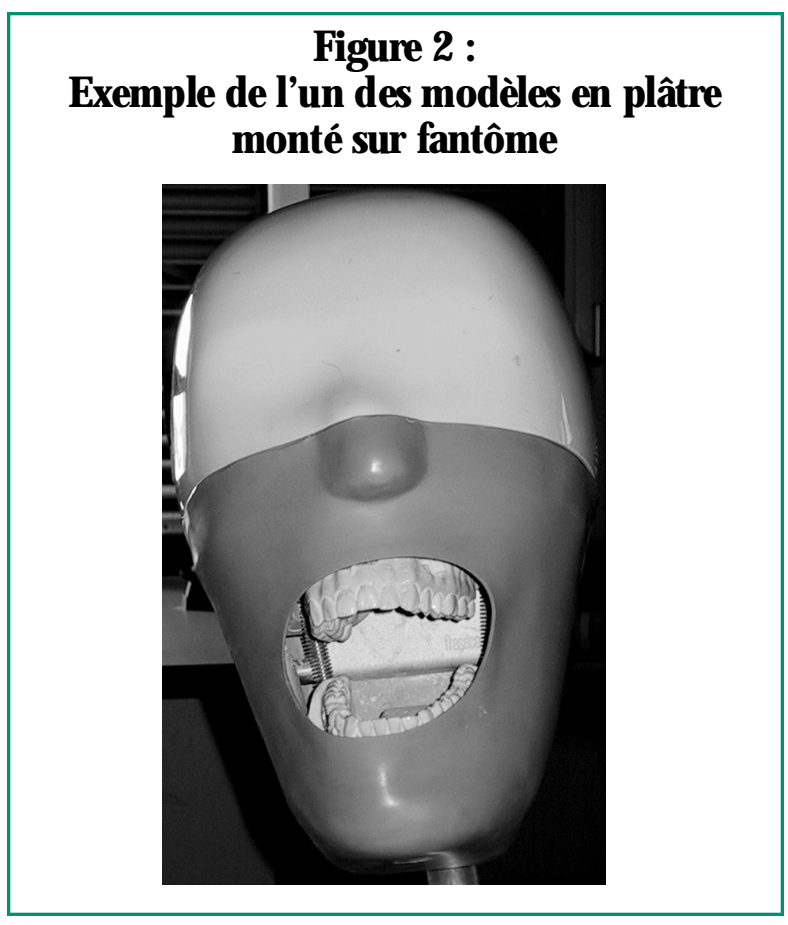

O $r$, nous avions demandéà nos apprentis de réaliser dès la deuxième séance de TP une cavité d'accès sur l'incisive supérieure les obligeant à travailler de cette manière. $\mathrm{C}$ et acte est difficile à effectuer : la moyenne des scores à I'issue de ceT $P$ a donc chuté. Avec le recul, nous réal isons que ce TP sur l'incisive supérieure arrivait trop tôt dans I'année $C$ ar, en plus dela découverte d'un nouveau maté riel (turbines), du travail sur dents naturelles et de la difficulté de la réalisation des cavités d'accès proprement dites, nous rajoutions aux étudiants une difficulté supplémentaire, celle de devoir travailler en vision indirecte. II reste important de noter le problème que posent les 


\section{Concepts et Innovations}

absents dans I'exploitation des résultats. Ces absents considérés comme val eurs manquantes compliquent l'interpré tation des résultats obtenus. L'étudiant absent à un TP se retrouve dans des conditions de travail différentes du reste de la promotion ; cette absence a également des consé quences sur l'autoévaluation. Une analyse différentielle des résultats obtenus par les étudiants absents à certaines séances de TP pourrait apporter des informations complémentaires.

Cetype de formation pratique a pour but de construireles bases des compétences endodontiques des étudiants de chirurgie dentaire. Le concept de compétence peut être abordé selon l'aspect structurel et dynamique. L'aspect structurel de la compétence est constitué de savoirs formalisés et de savoirs pratiques ${ }^{12,13}$. D 'une manière ou d'une autre, il sagit d'aider les étudiants à construire des schémas opératoires qui articulent à la fois des connaissances déclaratives et des connaissances d'action. Lorsque des habiletés psycho-motrices complexes sont visées, il est nécessaire de développer des stratégies d'apprentissage de type compilation, consistant à décomposer des connaissances relatives à des activités complexes en séquences plus simples (procéduralisation), afin de pouvoir les interpréter plus facilement, puis à induire une représentation plus globale (composition), en comparant sa performance à celle d'un modèl $e^{14}$. Pour ce faire, les enseignants doivent créer les conditions de développement de pratiques guidées, comportant auto-évaluation et feedback répété. Le dispositif de coévaluation que nous avons introduit nous semble aller dans ce sens.

Pour finir, Clark ${ }^{15}$ considère, à propos de la structure des études de deuxième cycle dentaire en Grande-Bretagne, que le modèle des triples cercles de $\mathrm{H}$ arden sadapte bien aux cinq premières années odontologiques. Ce modèle est similaire au modèle médical. II hiérarchise les acquisitions de connaissances et est basé sur les trois dimensions du travail d'un dentiste en tant que praticien compétent et réflexif. Ces dimensions concentriques sexposent en « ce que le dentiste est capable de faire » (faire le bon geste), « comment le dentiste appréhende sa pratique » (faire bien legeste) et enfin « le dentiste est un professionnel » (la bonne personne le fait). II pense que cette approche pédagogique en trois cercles concentriques fournit un outil capable d'encourager le développement de nouveaux concepts et d'améliorer les méthodes d'enseignement. II insiste sur le développement personnel des étudiants, qui résulte de connaissances désirées et sur le fait que le second cycle des études ne représente que la première étape de la formation continue que devrait suivre le praticien toute sa vie professionnelle.

Sur ces trois années d'étude, nous retiendrons que, grâceà ces séences, la maj orité des étudiantsa vu ses résultats progresser jusqu'à obtenir une compétence pratique initiale correcte. Cette compétence pratique se construit au fil des années d'études puis après grâce aux formations continues postuniversitaires.

\section{Conclusion}

Au début déroutant, le système d'autoévaluation que nous avons mis en place est devenu au fil des séances plus familier pour les étudiants. La plupart d'entre eux arrivent très vite à porter un regard critique sur leur travail. Parallèlement au développement de cette aptitude, on remarque une progression de la moyenne des scores des étudiants. M algréles limites de notreétude, il nous semble établi que la coévaluation de l'aménagement des voies d'accès endodontiques à partir de critères définis et objectivés visuellement a permis à un nombre significatif d'étudiants de développer plus rapidement un acte professionnellement satisfaisant. $M$ algré les contraintes qui existent et les difficultés qui devront être résolues en termes de logistique pédagogique, ces résultats, ainsi que l'accueil favorable par les étudiants de l'approche que nous avons développée, nous encouragent à envisager une utilisation plus systématique de ce processus de coévaluation dans notre enseignement.

\section{Remerciements}

$N$ ous tenons particulièrement à remercier le Pr Yves $M$ augars de la faculté de $M$ édecine de $N$ antes pour sa participation à la relecture de ce travail.

\section{Contributions}

Paul Calas et Franck Diemer ont adapté le dispositif pédagogique aux séances de travaux pratiques d'endodontie. Franck Diemer a participé à l'enseignement, effectué le recueil des données avec Elodie Prats, réal isé I'analyse statistique, élaboré la version initiale du manuscrit avec Marie-Eve Lauret et effectué les révisions finales. 


\section{Références}

1. Allal L. Versuneimplication activedel'élèvedansleprocessus d'évaluation formative Educateur $1984 ; 3$ : 22-26.

2. Bloom BS. Taxonomie des objectifs educatifs (vol 1 : domaine cognitif). Quebec: Presses de l'U niversité 1975 (traduction del'édition originale parue en 1956)

3. Campanale F. M odélisation des processus de l'autoévaluation en formation d'enseignants Pédagogies 1998 ; 12 : 13-19.

4. M anogue M , Brown GA, N attressBR, Fox K. Improving student learning in root canal treatment using self-assess ment. Int Endod J 1999 ; 32 : 397-405.

5. Scallon G. L'évaluation des apprentissages dans une approche par compétences Bruxelles: EditionsD e Boeck Université, 2004.

6. Perrenoud P. Touche pas à mon évaluation! Pour une approche systémique du changement. M esure et évaluation en éducation $1993 ; 16: 107-132$.

7. Paquay $L, D$ arras E, Saussez $F$ ( $C$ oord.). Les représentations de l'autoévaluation. In : Figari $G$ et Achouche M . L'acti vitéévaluative rénterrogée- Regards scolaireset socioprofessionnels. Bruxelles : Editions De Boeck Université, $2001: 119-133$.

8. De Peretti A, Boniface J, Legrand JA. Encydopédie de l'évaluation en formation et en éducation. Paris : EditionsESF, 1998.
9. M eirieu P. Pédagogie et évaluation différenciés In : D elorme C (Coord.) L'évaluation en questions. Paris : Editions ESF. 1987 : 149-165.

10. Belair LM. L'évaluation dans l'école Nouvelles pratiques Paris: EditionsESF, 1999.

11. Schön D . Le praticien réflexif. A la recherche du savoir caché dans l'agir professionnel. Montréal : Editions Logiques, 1983.

12. Mauban P. Rapports aux savoirs professionnels In : Chabchoub A (C oord.). Rapports aux savoi rset apprentissage des sciences Sfax : Publications de l'U niversité, 2000 : 111-119.

13. M alglaive $G$. Compétence et ingénierie de formation. In : Parlier M, M inet F et deW itteS (C oord.). La compétence, mythe, construction ou réalité? Paris: Editions l'H armattan, 1994 : 153-167.

14. Tardif J. Pour un ensaignement stratégique: L'apport de la psychologie coginitive. M ontréal(QC) : Les Editions Logiques 1992.

15. Clark JD, Robertson LJ, H arden RM . In our opinion: the first five years $A$ framework for dental undergraduate education. Br D ent J $2003 ; 195$ : 125-127.

M anuscrit reçu le 2 mars 2004 ; commentaires éditoriaux formulés aux auteurs le 12 mai 2004 ; accepté pour publication le 20 férrier 2005. 